\title{
La procaina intravenosa en los espasmos del cuello uterino
}

\author{
Por el doctor JORGE ORTIZ GAMBOA \\ Médico Residente del Servicio de Maternidad del Hospital \\ de San José de Bogotá
}

El cuello uterino cuando efectúa imperfectamente su proceso de dilatación puede ser en algunas circunstancias cousa específica de distocia. El segmento uterino inferior es relativamente inactivo en el parto normal y por lo tanto, hay un aumento gradual de actividad desde el fondo hacia abajo Si este tipo de contracción se invierte, es decir, si el segmento se hace hiperactivo, hay clerta disquinesia que provoca el paro del descenso de la presentación y también de la dilatación cervical.

En las primiparas añosas se observa con frecuencia un cuadro consistente en la detención de la dilatación del cuello uterino, de 2 a 4 cms., con borde resistente y rígido que se mantiene irreductible en el mismo estado. La mujer se queja de no tener momentos de reposo entre las contracclones, las "cuales resultan muy dolorosas. Esta rigidez espasmódica del orificio uterino es propla de mujeres nerviosas o que presentan alguna alteración en el sistema neurovegetativo.

Estos trastomos de la dínámica cervical no ceden siempre al empleo de espasmolíticos de uso corriente y tal fracaso parece ser debido en la mayoría de los casos a que la distocia tiene su raiz en una lesión casi orgánica de la fibra uterina o de su inervación cuando no a un estado de inestabilidad o susceptibilidad nerviosa.

Debido a estas perturbaciones del cuello uterino el parto puede prolongarse indefinidamente $y$ on caso de que tracasen los antiespasmódicos o para complementar su acción se han indicado algunas intervenciones como las incisiones del cuello y cuando no se logra la dilatación deseada recurrir a la cesárea segmentaria. En efecto, esta última, es la que decide la situación complicada por los trastomos de la dilatación uterina.

Administrando la Procaína intravenosa como analgesia en el parto, nos pudimos dar cuenta que en aquellas pacientes que presentaban la distocia cervical arriba descrita y el parto no se verificaba a pesan de las numerosas horas de trabajo y del empleo de espasmolíticos usuales, cedía tácilmente con esta droga sin alterar en lo más mínimo la marcha del trabajo del 
Wutte hil el estado de la maare o del feto, evitando con esto la práctica de intervenciones quirúrgicas como la cesárea que era el único recurso para terminar con estos partos prolongados.

Se hicieron varias observaciones, casi todas en primiparas añosas que presentaban esta distocia del cuello uterino, obteniendo un completo éxito en todos los casos, ya que cedieron sin que se hubiera presentado accidente alguno y las conclusiones a que pudimos llegar es el objeto de este estudio.

La Procaina fue sintetizada por Einhorn en 1905 e introducida bajo el nombre de novocaína. Es el más importante de los anestésicos locales y es destruida en el organismo más rápidamente que la cocaina.

Hasta 1941, las soluciones de Procaína fueron empleadas exclusivamente para anestesia local y raquidea. En este año, fueron publicados los primeros resultados de su uso intravenoso por Baurdín y Guiot, quienes la emplearon para combatir las crisis de asma bronquial con excelentes resulta. dos.

En 1942, Von Haecke, Briton y Guideaux publicaron informaciones sobre los resultados obtenidos con su em. pleo intravenoso para prevenir los accidentes de la arseno-intolerancia. Lundy, ya había tenido algunas experencias sobre iesultacios satisíactorios en numerosos casos de ictericia crónjca para aliviar el prurito.

En 1943, Leriche publicó sus observaciones sobre los resultados muy halagadores en la enfermedad de Ray. naud ya que alivia el dolor $y$ disminuye los trastornos vaso-motores. Gor- aon, en el Lanaaa, la empleo después en la sedación completa en varios casos de quemaduras, sus acciones fue ron sostenidas cerca de doce horas.

En 1945, Mc Lachlin empleó las soluciones de Procaína, vía endovenosa en los post-operatorios, en reemplazo de la morfina. Allen, la utilizó como analgésico obstétrico durante el tercer período del trabajo obteniendo dos grados de analgesia.

En 1946, del Río la usó en 100 casos de quemaduras extensas. Longo y Sosa Gallardo, de la Argentina, la emplearon en el infarto del mesenterio. State y Wagensteen en el shock anafiláctico. En 1948, Bitrich y Powers, obtuvieron los primeros resultados como anestésico general en cirugia.

En Colombia se comenzó a emplear en el año de 1950. El doctor Mesa Upegui, de Medellín, la utilizó como analgésico obstétrico durante el período expulsivo y como anestésico en algunas intervenciones obstétricas. Luego fue empleada como anestésico general en cirugía asociada al Pentothal, como tratamiento en los ataques de eclampsia y se continuó aplicando como analgésico obstétrico en el Servicio de Maternidad del Hospital de San José.

La Procaina, según la teoría más aceptada, en concentraciones bajas en la sangre no produce anestesia local en los tejidos sanos, sino en aquellos de regiones afectadas que son dolorosos, inflamatorios o edematosos. El estado de permeabilidad capilar permite que la Procaína vía endovenosa penetre en los tejidos y anestesie los nervios que alli terminan interviniendo en el arco reflejo que origina el dolor, los espasmos y el edema. 
th los espasmos dei cuenu wetho los tejidos de esa región se tornan edo matosos y congestionados cumpliendo así con el aumento de la permeabilidad capilar y por lo tonto presentan todas las cualidades para que la Pro caina administrada vía endovenosa, vaya a actuar -ápida y profundamen. to en las terminaciones nerviosas, cediendo con esto el espasmo cervical. sin alterar el ritmo de las contracciones uterinas y la marcha del trabajo del parto.

Todas las observaciones fueron realizadas en el Servicio de Maternidad del Hospital de San Jasé y se escogieron pacientes casi todas primiparas. cuyas edades oscilaron entre 27 y 43 años y que presentaban esta distocia del cuello uterino. Con el fin de obtener conclusiones mejores se dejaba que el trabajo tuviese un tiempo máximo de duración controlando el estado general de la enferma y los latidos fotales. Durante esas horas se administraban antiespasmódicos corrientes $y$ una vez que no producia efecto alguno se procedía a la aplicación de la Procaína, vía intravenosa.

Se utilizó Procaína hidrochloride cristalina, distribuida por The NorthStrong Corp. de Washington. Se presenta en frascos de 5 grs., con tapón perforable y completamente estéril.

Para preparar la solución al $1 \%$ se mezcia el contenido de cada trasco en 500 c. c. de dextrosa al $5 \%$ en agua destilada. La vena punción, con aguja $N^{9} 20$, se hace en la flexura del codo o en el antebrazo y se debe preferir la región del antebrazo ya que permile ciertos movimientos sin peligro de des. plazar la aguja. La inyección se efec-

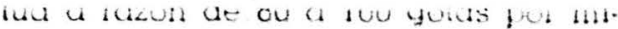
nuto y su aplicación se debe hacer on un promedio de una hora a una hora y media de duración para los 500 c. c. de la solución.

Antes de iniciar la inyección, la pa. ciente debe ser trasladada a la sala de partos e instalada en la mesa con todos los requisitos para poder vigi. larla permanentemente controlando el pulso, la tensión arterial, respiración y los ruidos cardíacos fetales.

Una vez que todo está listo se pro cede a la aplicación de la solución de Procaína. Se comienza lentamente y luégo se va aumentando la velocidad de la inyección de un modo gradual. A medida que esto sucede, se pueden presentar ligeros signos subjetivos como marecs, inquietud $y$ excitación. Aumentando la dosis, la enferma queda profundamente dormida, no se da cuenta de los dolores, a veces se que ja ligeramente con cada contracción.

Se pudo comprobar que esta droga administrada vía endovenosa no ejerce acción nociva alguna sobre el organismo materno o fetal. Se puede presentar taquicardia que cede inmediatamente que termina su oplicación. La tensión arterial no sufre ninguna modificación. La respiración es amplia y tranquila. Algunas pacientes tienen vómitos cuando han ingerido alimentos momentos antes de la inyección. Las funciones renales permanecen normales, hay aumento de la cantidad de orina tal vez debido a la dilatación de los vasos renales y no ha habido casos de incontinencia de orina o de relenctón vesical.

Sobre el sistema nervioso; al comienzo puede haber confusión mental 
y conversacion lenta, luego, tanto en pacientes agitadas como en las calmadas, produce un sueño tranquilo.

Las contracciones uterinas no sufren modificación, continúan presentándose intermitentes, involuntarias y además indoloras. Siguen siendo efectivas y de buena intensidad caracterizándose por no ocasionar molestias a la pa. ciente.

La Procaina endovenosa acelera la marcha del trabajo del parto y en aquellos, prolongados por espasmo del cuello uterino, hace aumentar la dila. tación cervical y, los cuellos edema. tosos, duros y resistentes, se vuelven blandos llegando a la dilatación completa en pocos minutos. La misma re lajación se obtiene en los tejidos blandos y permite que sean fácilmente dilatables por la presentación sin oponer mayor resistencia.

En la cavidad uterina no es perju. dicial para el feto, sin embargo, en algunos casos se observaron signos de sufrimiento fetal que fueron mejoran do a medida que avanzaba el periodo indoloro del trabajo $y$ en otros, se atribuyeron al trabajo prolongado de las pacientes y no por acción tóxica de la droga. Por este motivo la mayo. ría de los fetos fueron extraídos. por medio del fórceps, ya que las enlermas llegaban a la sala de partos en marcado estado de agotamiento físico, debido al trabajo prolongado a que se sometion $y$, los esfuerzos re queridos para la expulsión fetal eran insuficientes.

El alumbramiento se realiza dentro de los límites normales, no se han presentado retenciones placentarias. La Procaína no altera en nada la evolución y funclonamiento de los órga- nos de la madre en el puerperio. El desarrollo ulterior de los niños no es afectado y todos salieron en buen estado del hospital.

Como manifestaciones de intolerancia que se pueden presentar aplicando la Procaína endovenosa en solución al $1 \%$, están los trastornos respi. ratorios, cianosis, que ceden a la aplicación de algún analéptico y al oxi. geno inhalado; la apnea no se puede considerar como signo de sobredo sificación, pues ocurre al mismo tiem. po que el colapso circulatorio y el mo jor tratamiento consiste en insuflar oxígeno. A veces se observan signos de irritabilidad motora que no ofrecen mayor peligro como son las contracciones musculares generalizadas, mo vimientos convulsivos que están precedidos de espasmos de los músculos faciales y por regla general cesan al disminuír la velocidad de la venoclisis debido a la rápida absorción y eliminación de la Procaina en el hígado. Todas esas mantfestaciones pueden evitarse administrando una cópsula de Seconal media hora antes de iniclar la inyección de la solución y cuando se presentan desaparecen rápidamente al inyectar barbitúricos, vía endovenosa.

Así pues, en la totalidad de los casos de espasmos del cuello uterino en que se aplicó la Procaina vía endovenosa, se obtuvo un resultado excelente ya que todos cedieron sin que se hubiera presentado alguna consecuencia fatal desagradable después de su administractón, debido a que la Procaína circulante en el torrente sanguíneo está siempre bajo el dominio del operador. En efecto, puesto que es destruida en pocos minutos, y como la in- 
yección puede ser iniciada con lentitud, aumentada con cuidado $e$ interrumpida al primer signo de peligro, es evidente que el uso endovenoso de la Procaína ofrece una ventaja definida sobre los métodos en los cuales no puede regularse la tasa de absor. ción como es el caso de la infiltración local y de la anestesia raquídea, porque el agente anestésico una vez in. yectado no se puede controlar.

A continuación describiremos algunas de las observaciones realizadas con el empleo de la Procaína endovenosa para los espasmos del cuello uterino y en seguida las conclusiones que se pudieron obtener.

\section{Caso número 1}

Primigestante a término de 28 años. Presentación cefálica de vértice. Ingresa al hospital a las $12 \mathrm{~m}$. del 25 de junio. Siete horas antes habia iniciado el trabajo de parto espontánea. mente. Contracciones regulares y de muy buena intensidad. Al examen vaginal se encuentra un cuello blando, borrado, dilatación de $1 \mathrm{~cm}$., membranas intactas. Se ordena enema evacuador y se deja en observación. Treinta horas más tarde se vuelve a examinar y la dilatación cervical, tan sólo ha llegado a $2 \mathrm{cms}$., se halla borado el cuello pero ha tomado una consistencia dura. Se prescribe una ampolleta de Atrinal. A las 11 p. m. del 26 de junio las contracciones uterinas se repiten cada 7 minutos, con duractón de 50 segundos y muy dolorosas. Se ordena una cápsula de Seconal. Los ruldos fetales son normales. Después de sesenta horas de estar en trabajo de parto intenso, la dilatación del cue llo uterino no ha pasado de los $2 \mathrm{cms}$. a pesar de la aplicación de antiespasmódicos y sedantes. Se aplica entonces los 500 c. c. de la solución de Procaina al $1 \%$, via endovenosa a razón de 80 gotas por minuto. A los diez minutos, la enferma duerme profundamente, las contracciones siguen su ritmo anterior $\sin$ ocasionar dolor alguno; los ruidos fetales son normales. E! total de la solución se aplica en un tiempo de una hora y quince minutos sin que se haya presentado reacción alguna por parte de la madre o del feto. Se hace nuevo examen vaginal encontrándose un cuello blando, bo rrado, $8 \mathrm{cms}$. de dilatación. Las membranas se rompen artificialmente. Minutos más tarde la dilatación es completa. Se aplica fórceps profiláctico y se obtiene un feto vivo que respira espontáneamente y pesa 3.100 grs. El alumbramiento se hace 15 minutos después. El puerperio es normal. La enferma sale a los siete días del hospital por mejoría. Niño sano.

\section{Caso número 2}

Secundigestante a término de 34 años. Presentación cefálica de vértice. Antecedentes obstétricos: un aborto de cinco meses. Llega al hospital veinte horas después de haber comenzado tarabajo de parto espontáneamente. Las contracciones uterinas han sido frecuentes $y$ de buena intensidad. Al tacto vaginal se aprecia un cuello borsado, delgado, duro, $2 \mathrm{cms}$. de lilatación y membranas intactas. Se prescribe una ampolleta de Atrinal y un enema evacuador. La paciente continúa en trabajo durante todo el día, las contracclones uterinas le ocasionan in. tenso dolor y se suceden cada dos minutos con una duración de 50 segun- 
dos. Nuevo examen vaginal revela un cuello edematoso, duro, grueso, dilatación de $5 \mathrm{cms}$. y membranas íntegras. El trabajo ha tenido una duración de 44 horas, la enferma se encuentra muy agotada y los dolores se han hecho insoportables. Se comienza a plicar la solución de Procaina al $1 \%$ vía endovenosa a razón de 80 gotas por minuto. La enterma duerme profundamen. te, las contracciones uterinas continúan con el mismo ritmo anterior. Los ruidos fetales no se ham alterado. Treinta mlnutos más tarde presenta espasmos musculares, convulsiones y vómitos que ceden tan pronto como se suspen¿de la inyección. Después se reanuda con la misma velocidad y se terminan los 500 c. c. sin que nuevas reacciones se presenten ni se haya alterado el trabajo del parto. Al tacto vaginal se encuentra un cuello blando, borrado, delgado, dilatación de $9 \mathrm{cms}$., se rompen artificialmente las membranas y media hora después la dilatación es completa. Se hace aplicación de fórceps previa episiotomía. El alumbramiento se hace 5 minutos más tarde. El puerperio es normal.

\section{Caso número 3}

Primigestante a término de 27 años. Feto único en presentación cefálica de vértice. El trabajo de parto se inició a las 2 a. m. del 7 de agosto. La pacien. ta iba a ser atendida en el parto por una comadrona. En las horas de la noche las contracciones uterinas eran muy frecuentes y le ocasionaban intenso dolor. La comadrona le practicó varios tactos vaginales y se dió cuenta que la dilatación cervical se había detenido $y$ en cambio los dolores se hacian insoportables para la paciente y por este motivo decidieron hospitalizarla. Ingresó a las 11 p. m., se hallaba muy excitada, emitía grandes gritos con cada contracción pidiendo que la operaran y le extrajeran el niño. Las contracciones tenían una duración de 45 segundos y se repetían cada $2 \mathrm{mi}$ nutos. A.l tacto vaginal practicado a las 11.30 p. m., 21 horas después de haber comenzado trabajo, se encontró un cuello grueso, edematoso y duro, renía una dilatación de $5 \mathrm{cms}$., membranas rotas y presentación encajada. Los genitales externos se hallaban edematosos y los tejidos vecinos muy congestionados. Ruidos fetales velados. A las $11.45 \mathrm{p} . \mathrm{m}$. se inició la aplicación de la solución de Procaína al $1 \%$. Minutos más tarde la enferma entró en un período de somnolencia, sólo se quejaba ligeramente con cada contracción y luégo dormía profundamente. El trabajo no se alteró. Presentó después espasmos musculares generaliza. dos y cianosis de la cara y extremida. des. Se suspendió temporalmente la inyección y se administró oxígeno in. halado. La paciente se despertó rápidamente y cesaron esas manifestaciones. Se reanudó la aplicación de la Procaína hasta completar los 500 c. c. Los ruidos fetales eran normales. La enferma se fue despertando lentamente. Al examen vaginal se encontró un cuello en dilatación completa; se hizo onlicación de fórceps y se obtuvo un felo vivo de 3.000 gramos que resplró espontáneamente. No hubo complicación alguna en el puerperio.

\section{Cosso número 4}

Primigestante a término de 43 años. Presentación celálica de vértice. Emba. razo de evoluctón normal. Ingresa al 
hospital a las 9 a. m. del 30 de agosto después de nueve horas de haber iniciado trabajo de parto espontánea. mente. Las contracciones uterinas tienen una trecuencia de 8 minutos con una duración de 35 segundos. Ruidos fetales en buen estado. Al tacto vaginal so encumtra un cuello bo:rado. delgado algo duro dilatación de 2 cms. y membranas intactas. Durante todo el día la paciente se halla en el mismo estado, las contracciones continúan regulares y de buena intensidad. Al día siguiente, 3 p. m., treinta horas después de haber comenzado trabajo, se hace examen vaginal nuevamente y se encuentra un cuello borrado, duro, dilatación de $4 \mathrm{cms}$. y membranas intactas. Las contracciones vienen cada cinco minutos $y$ tienen una duración de 30 segundos. Se ordenan dos ampolletas de Atrinal y una cápsula de Seconal. A las 10 p. m. las contracciones siguen con la misma frecuencia $\theta$ intensidad, las membranas se rompen artificialmente. A las 6 a. $\mathrm{m}$. del día siguiente la enferma se halla muy agotada, lleva 43 horas de trabajo. Los ruidos fetales son poco frecuentes y velados. El tacto vaginal deja percibir un cuello grueso, edematoso, grueso con la misma dilatación de $4 \mathrm{cms}$. que tenía el día anterior y que no había aumentado a pesar de la aplicación de los antiespasmódicos. Se inicia entonces la colicación de la Procaína al $1 \%$ vía endovenosa habiendo dado media hora antes una cápsula de Seconal. Durante la inyección de los 500 c. c. no se presenta reacción alguna de intolerancia por la droga y la enferma duerme tranquila. mente. Las contracciones uterinas continúan con el ritmo anterior. Los rul- dos fetales han mejorado. El total de la solución se aplica en una hora: una vez terminada se hace examen vaginai $y$ se encuentra el cuello uterino en dilatación completa, las mem. branas rotas y la presentación enca. jada. Se hace aplicación de fórceps $y$ se obtiene un feto de 2.700 gramos que respira inmediatamente. El alumbramiento se produce i0 minutos des. pués. Hay ligera hipertension postalumbramiento. Puerperio normal.

\section{Caso número 5}

Primigestante a término de 38 años. Altura uterina $36 \mathrm{cms}$. Presentación cefálica de vértice. Ruidos fetales positivos. Llega al servicio doce horas después de haber comenzado trabajo. Las contracciones uterinas se repetian cada dos minutos con una duración de 42 segundos. Al tacto vaginal se encuentra un cuello borrado, duro, resistente, con una dilatación de $2 \mathrm{cms}$. membranas intactas. Se prescriben antiespasmódicos de tipo Atrinal y después Tolserol vía endovenosa. Continúa en trabajo 24 horas más; lleva en total 42 horas; los antiespasmódicos aplicados no han hecho electo ya que la dilatación cervical tan sólo ha llegado a 3 cms. el cuello se halla duro, edematoso. Para terminar con este traba. jo prolongado se aplica la solución de Procaina al $1 \%$ vía endovenosa pre via ingestión de una cápsula de $\mathrm{S} \theta$ conal. A las 5.30 p. m. las contracciones vienen cada 2 minutos $y$ t tienen una duración de 45 segundos, son muy dolorosas; a las 6 p. m. se da una cáp. sula de Seconal. A las 6.45 p. m. se Inicia la aplicación de la inyección a tazón de 100 gotas por minuto, 8.15 p. $m$. se termina sin que se haya pre- 
a.guna. y.ju p. m. tacto vaginal: cuello blando, borrado, delgado, dilatación de $9 \mathrm{cms} .9 .45 \mathrm{p}$. m. se rompen artificialmente las membranos. 9.50 p. m. expulsión espontánea de un feto que respira inmediatamente y pesa 3.145 gramos. El alumbramiento se hace 20 minutos más tarde. Sale de la sala en buenas con diciones. Puerperio normal.

\section{Caso número 6}

Secundigestante a término de 32 años. Antecedentes obstétricos: Un aborto de dos meses. Presentación cefálica de vértice. Ingresa al servicio 12 horas después de haber comenzado trabajo espontáneamente. Las contracciones uierinas son frecuentes y de buena inlensidad. El cuello uterino se encuentra borrado, blando y comienza a dilatarse. Ruidus fetales normales. Durante las 24 horas siguientes la paciente continúa en el mismo estado, el cuello tan sólo ha alcanzado una dilatación de dos centímetros. Se aplica una ampolleta de Atrinal, vía intramuscular $y$ se sigue observando. Después de 68 horas de trabajo, durante el cual las contracciones uterinas se han venido repitiendo cada cinco a ocho minutos, con una duración de 45 a 60 segundos, la dilatación cervical se halla aún en dos centímetros. El trabajo del parto parece prolongarse indefinidamente. La enferma se encuentra muy agotada. No resiste los dolores que le ocasionan las contracclones uterinas. Los ruidos fetales son normales. Se procede entonces a aplicar la Procaina, vía endovenosa, a razón de cien gotas por minuto, 500 c. c. en total de la solución al $1 \%$. So aplica en un tiempo de hora y media,
Ia enterma duerme profunáamente y no presenta reacción alguna. El traba. jo de parto no ha tenido modificación en cuanto se refiere a la frecuencia $\epsilon$ intensidad de las contracciones uterinas. Se hace tacto vaginal y se en. cuentra un cuello blando borrado, con una dilatación de $9 \mathrm{cms}$., las membra nas se rompen artificialmente. Minutos más tarde la dilatación es completa. Se hace aplicación de forceps, ya quo los ruidos fetales se hallan velados $y$ el líquido amniótico sale con meconio. Se obtiene un feto de 3.300 gramos que respira espontáneamente. El alumbramiento se verifica 25 minutos des. pués. Hay un desgarro de primèr grado que se sutura. El puerperio fue normal y la sutura prendió.

\section{Caso número 7}

Primigestante a término de 27 años. Presentación cefálica de vértice encajada. Ingresa al Hospital a las 10 a. m. del 19 de julio, había comenzado trabajo a las 2 a. m. de ese día. Las contracciones uterinas se verificaban cada 7 minutos con una duración de 45 segundos. Se hace tacto vaginal $\gamma$ se encuentra un cuello borrado con una dilatación de $5 \mathrm{cms}$., membranas intactas. El trabaio continúa el ritmo anterior, pero las contracciones uterinas son muy dolorosas. La paciente sigue en las mismas condiciones hasta el dia siguiente, a la 1 p. m. se hace nuevo tacto vaginal, el cuello uterino se halla muy edematoso, duro, con los mismos 5 cms. de dilatación y las membranas rolas. La enferma lleva 35 horas de trabajo: el cuello se ha detenido en su dilatación: se procede entonces a aplicar la solución de Pro. caina al $1 \%$, vía endovenosa, a ra- 
zon de 80 gotas por minuto. Tan sólo se inyectan 250 c. c. de la solución ya que la enferma presenta convulsiones y vómito. Una hora más tarde se hace nuevo tacto vaginal, encontrando el cuello en dilatación completa. So aplica foreps y se extrae un feto de 3.207 gramos de asperto nomal y que I.si in espontinenmente. El alumbranirnto se hace 5 minutos después. Hoy un desgarro perineal de primer grado que se sutura.

\section{Caso número 8}

Primigestante a término de 32 años. Presentación O. I. A. Comenzó trabajo espontáneamente a las 2 a. $\mathrm{m}$. del 11 de junio. 24 horas más tarde el cuello uterino se encontraba borrado, algo arueso, de consistencia dura y una dilatación de $2 \mathrm{cms}$. Continuó la enferma en el mismo estado durante el dia siguiente y se le aplicó Atrinal, vía intramuscular. Ya llevaba 43 horas en trabajo, durante el cual las contracciones se realizaban regularmente y con muy buena intensidad. El cuello ute. rino se hallaba en $4 \mathrm{cms}$. de dilatación y tenía una consistencia dura. A las 9 y 30 p. m. del 12 de junio. con 45 horas de trabajo, la dilatación no había aumentado nada en el curso de las últimas 10 horas. Se administró entonces 500 c. c. de la solución de Procaína al $1 \%$ en un tiempo de 1 hora y 40 minutos, sin que hubiera reacción alguna por parte de la enferma. Se hizo nuevo tacto vaginal y se encuentra un cuello de consistencia blanda, con una dilatación de $9 \mathrm{cms}$. Media hora más tarde la dilatación es completa. Se hace aplicación de fórceps y se obtlene un feto de $3.440 \mathrm{gra}$ mos que respira espontóneamente. El alumbramiento tuvo lugar 20 minutos más tarde. Puerperio normal.

\section{Caso número 9}

Primigestante a término de 30 años. Presentación celálica de vértice. Ingresa la enterma al Hospital después de 18 horas de haber comenzado trabajo espontaneamente. Las contracciones son regulares, de buena intensidad $y$ duración. Al tacto vaginal se aprecia un cuello largo, blando, sin dilatación alguna. Se deja que continúe trabajando durante todo el día ya que los ruidos fetales se hallom en buenas condiciones. Al día siguiente, a pesar de que las contracciones uterinas se repiten cada cinco minutos, con una duración de 50 segundos, el cuello no presenta dilatación. La enferma lleva 46 horas de trabajo intenso; el cuello uterino continúa cerrado. Se hace la aplicación de 500 c. c. de solución de Procaina al $1 \%$ vía endovenosa, gota a gota, gastando solamente una hora $\sin$ que se presente manifestación de intolerancia por parte de la madre. El trabajo continúa su ritmo anterior. Los ruidos tetales no se han modificado. Duerme profundamente durante ese tiempo, a cada contracción se queja débilmente. Terminados los 500 c. c. de la solución de Procaina la enferma despierta rápidamente. Se hace tacto vaginal y se encuentra un cuello blando borrado, con una dilatación de $6 \mathrm{~cm}$. y las membranas intactas. Se aplican dos unidades de Pitocin vía intramuscular. Hora y media más tarde la dilatación cervical es completa y se ve. rifica ta expulsión del feto expontá. neamente. Hay un desgarro perineal de primer grado que se sutura. El toto de aspecto normal, pesa 2.900 grs. 
y respira espontuneanente. L: cumbramiento se hace 10 minutos más tarde. Hay ligera hemorragia. Puerperio normal.

\section{Caso número 10}

Primigestante a término de 28 años. Presentación cefálica de vertice enca. ada. Feto único. Inicio rabajo a las 5 a. m. del $1^{\circ}$ de actubre. Contraccio. nes regulares y de buena intensidad: 15 horas más tarde el cuello uterino sólo presenta $1 \mathrm{~cm}$. de dilatación. Continúa en trabajo 24 horas más. La dilatación llega a $6 \mathrm{~cm}$. y alli se estaciona. El 3 de octubre el trabajo de parto no ha progresado, el cuello uterino de consistencia dura sigue con 6 $\mathrm{cm}$. de dilatación. Se han administrado antiespasmódicos $\sin$ resultado. Las contracciones son buenas en intensidad y frecuencia, pero el trabajo tiende a prolongarse indefinidamente; entonces se aplica la solución de Procaina al $1 \%$ endovenosa. A las 8 a. m. se da una cápsula de Seconal. $A$ las 8 y 40 a. m., se inicia la inyección a razón de 80 gotas por minuto: 9 y 10 a. m., se aplican 2 unidades de Pitocin intramuscular; 10 y 10 a. m. se terminan los 500 c. $c$ de solución en un tiempo de hora y media. El suello uterino se encuentra en dilatación completa. A las 11 y 15 a. $\mathrm{m}$. se realiza la expulsión espontánea de un feto que pesa 2.560 grs. y que respira inmediatamente. A las 11 y 30 a. m. un alum. bramiento espontáneo. Puerperio normal.

\section{CONCLUSIONES}

$1^{\text {a }}$ Se describe un cuadro que se presenta con trecuencia en las primiparas añosas y que consiste en la do- tencion de la anatacion del cuello uterino durante el trabaio del parto.

$2^{a}$ Esa rigidez espasmódica del orificio uterino cede fácilmente a la administración de 500 c. c. de solución de Procaina al $1 \%$ vía endovenosa.

$3^{\circ}$ Se resumen diez observaciones realizadas en pacientes, casi todas pri. miparas cuyas edades oscilaron entre 27 y 43 años, que presentaban esa dis. tocia del cuello uterino y que cedió fácilmente con la aplicación de la Procaina, vía endovenosa.

$4^{a}$ Se hace un pequeño recuento histórico del empleo de esta droga y sus principales indicaciones administrada, vía endovenosa.

$5^{a}$ Se comprueba que la Procaina aplicada en esta forma no produce anestesia local en los tejidos sanos, sino en aquellos que son dolorosos, inflamatorios y edematosos ya que el aumento de la permeabilidad capilar permite que la Procaina penetre en los tejidos y anestesie los nervios que allí terminan.

$6^{\circ}$ En los espasmos del cuello uterino los tejidos se tornan edematosos, dolorosos y congestionados, cumplien. do así con el aumento de la permeabilidad capilar y por lo tanto presentan todas las cualidades para que la Procaína administrada vía endovenosa, vaya a-actuar sobre las terminaciones nerviosas finalizando con el espasmo cervical sin alterar la marcha del tratrabajo del parto.

$7^{a}$ Se describe la manera de preparar las soluciones de Procaína, la técnica de su aplicación y los cuidados que se deben observar para poder controlar el estado de la madre y del feto durante la inyeccióni con el fin de 
actuar inmediatamente que se presente alguna manifestación de intolerancia por la droga.

$8^{a}$ Se comprueba que la Procaína endovenosa no es nociva para la madre o el feto y además no modifica el ritmo, duración e intensidad de las contracciones uterinas.

9: Por las observaciones realizadas se puede concluir que la Procaina apli. cada vía endovenosa es el medicamento ideal para el tratamiento de los espasmos del cuello uterino por su efecto comprobado en la totalidad de los casos en que se empleó, por su inocuidad tanto en la madre como en el feto, por no alterar la marcha del trabajo del parto, del período de expulsión fetal, del alumbramiento, de la evolución del puerperio y del desarro110 del niño en los días siguientes. Además por la facilidad y comodidad de su aplicación, y por último, por el mínimo de manifestaciones de intolerancia que se pueden presentar y que ceden inmediatamente que se suspende su administración o la velocidad de la inyección, en ningún caso son peligrosas y se pueden prevenir por medio de la ingestión de una cápsula de Seconal media hora antes de ini. ciar su aplicación.

10. Por esta propiedad de vencer los espasmos del cuello uterino, especialmente en las primíparas añosas, hace que se acorte de una manera conside rable la duración del trabajo del parto que de otra manera se necesitarían largas horas para poder salvar ese obstáculo o someter a las pacientes a intervenciones qulrúrgicas ya que dejándolas abandonadas a sus pro

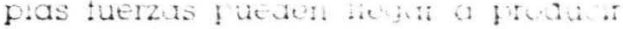
una inercia secundaria, una ruplura uterina con sus consecuencias o la muerte del feto en el útero.

\section{BIBLIOGRAFIA}

\section{Allen. F. M.-Intravenous Obstetrical Anesthosic. Amer. J. Surg. 70:283. diciembre 1945 .}

Gordon. R. A.--Intravenous Novocaine for Analgesia in Burns. Canad. Med. Assn. J. 49:479, diciembre, 1943.

Fraser, R. J.-Analgesia con Pentothal-Procaina endovenosa. Anesth. y Analg. 27:159, mayo-junio, 1948.

Gilbert, C. R. A.-Intravenous Procaine for Obstetrical Anesthesia. Anesth. y Analg. 25:133, "iulio-agosto, 1946.

Belinkoty, S.-Intravenous ProcainePentothal during Labor. Amer. J. Obst. \& Ginec. 62:1129, noviembre, 1951.

Trujillo. J. H.-Anestesia General en Cirugía por medio de la Procaína Endovenosa. Tesis de Grado. 1950.

Gilman. S.- The Treatment of Dan. geruo Reactions to Novocaine. New England, 1. Med. 219:841, noviem. bre, 1938.

Mc. Lachlin. J. A.-The Intravenous Procaine Use as a Substitule for Morphine in Postoperative Care. Cand. Med. Assn. 52.383, abril, 1945.

Mesa Upegui, D.-Analgesis Obstétrica durante el período expulsivo y Anestesia en algunas intervenciones quirúrgico-obstétricas por Novocaína endovenosa. Tesis de Grado. 1950. Allen, F. M.--Intravenous Procaine Analgesia. Anesth y Analg. 25:1, enero-febrero, 1946. 